\title{
Liver and spleen biometrics in childhood-onset systemic lupus erythematosus patients
}

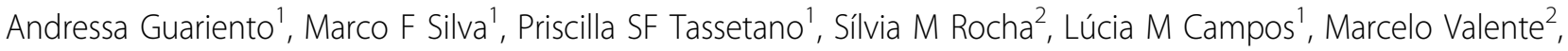 \\ Clovis A Silva ${ }^{*}$
}

From 21st European Pediatric Rheumatology (PReS) Congress

Belgrade, Serbia. 17-21 September 2014

\section{Introduction}

Involvement of the reticuloendothelial system occurs in 20-50\% childhood-onset systemic lupus erythematosus (c-SLE) patients at disease onset, usually associated with disease activity. Hepatomegaly and/or splenomegaly may also be associated with abnormal liver function tests. Abdominal ultrasound can be used to assess liver and spleen measurements in children and adolescents without risk of radiation. However, a systematic evaluation of these visceral organ dimensions has not been performed in C-SLE population, particularly during the disease course.

\section{Objectives}

To evaluate liver and spleen dimensions in c-SLE patients and healthy controls and to assess possible associations between abnormalities in liver and spleen sizes with demographic data, clinical features, disease activity, cumulative damage and treatment.

\section{Methods}

30 c-SLE patients and 30 healthy control volunteers underwent abdominal ultrasound. The following two liver measurements were performed in left hepatic lobe: craniocaudal and anteroposterior and three in right hepatic lobe (RHL): posterior craniocaudal (PCC-RHL), anterior craniocaudal and anteroposterior. Three spleen dimension measurements were also evaluated: longitudinal, transverse and anteroposterior. Demographic, clinical and laboratorial data, and treatment were assessed. Disease activity was evaluated according to SLE Disease Activity Index 2000 (SLEDAI-2K), European Consensus Lupus Activity

'Pediatric Rheumatology Division, Faculdade De Medicina Da Universidade De São Paulo, São Paulo - SP, Brazil

Full list of author information is available at the end of the article
Measurement (ECLAM) and Systemic Lupus Activity Measure (SLAM) scores.

\section{Results}

Mean current age was similar in c-SLE and controls $(170.31 \pm 27.81$ vs. $164.15 \pm 39.25$ months; $\mathrm{p}=0.486)$, likewise the frequency of female gender $(77 \%$ vs. $63 \%$, $\mathrm{p}=0.398$ ). The mean of PCC-RHL dimension was significantly higher in C-SLE compared to controls (13.30 \pm 1.85 vs. $12.52 \pm 0.93, p=0.044)$. There were no differences between the other hepatic biometrics and splenic parameters $(p>0.05)$. Further analysis in c-SLE patients according to PCC-RHL dimension $>13.3 \mathrm{~cm}$ (mean of this biometric measurement in $30 \mathrm{c}-\mathrm{SLE}$ patients) versus < $13.3 \mathrm{~cm}$ showed that the median of SLEDAI-2K [8 (0-18) vs. $2(0-8), \mathrm{p}=0.004]$, ECLAM [ $4(0-9)$ vs. $2(0-5), \mathrm{p}=0.019]$ and SLAM [5 (1-13) vs. $2(0-14), p=0.016]$ were significantly higher in patients with higher PCC-RHL dimension, likewise the mean of erythrocyte sedimentation rate (33.7 \pm 16 vs. $22.0 \pm 13 \mathrm{~mm} / 1^{\text {st }}$ hour, $\mathrm{p}=0.038$ ). The frequencies of nephritis were significantly higher in patients with PCC-RHL dimension $>13.3 \mathrm{~cm}$ versus $<13.3 \mathrm{~cm}$ (77\% vs. $29 \%, p=0.010)$. The median of serum liver enzymes were similar in both groups $(\mathrm{p}>0.05)$. Positive correlation was observed between SLEDAI- $2 \mathrm{~K}$ and PCC-RHL $(\mathrm{p}=0.001$, $r=+0.595)$. Negative correlation was evidenced between disease duration and longitudinal dimension of spleen $(\mathrm{p}=0.031, \mathrm{r}=-0.394)$.

\section{Conclusion}

Our data raises the novel possibility that disease activity could lead to a subclinical and localized hepatomegaly during the disease course. Long disease duration resulted to spleen atrophy in c-SLE patients. 


\section{Disclosure of interest}

A. Guariento: None declared, M. F. Silva: None declared, P. S. F. Tassetano: None declared, S. M. Rocha: None declared, L. M. Campos: None declared, M. Valente: None declared, C. Silva Grant / Research Support from: This study was supported by Fundação de Amparo à Pesquisa do Estado de São Paulo (FAPESP - grants 2008/58238-4 to CAS), by Conselho Nacional do Desenvolvimento Científico e Tecnológico (CNPQ grant 302724/2011-7 to CAS), by Federico Foundation to CAS and by Núcleo de Apoio à Pesquisa "Saúde da Criança e do Adolescente" da USP (NAP-CriAd).

\section{Authors' details}

${ }^{1}$ Pediatric Rheumatology Division, Faculdade De Medicina Da Universidade De São Paulo, São Paulo - SP, Brazil. ²Pediatric Radiology Department, Faculdade De Medicina Da Universidade De São Paulo, São Paulo - SP, Brazil.

Published: 17 September 2014 